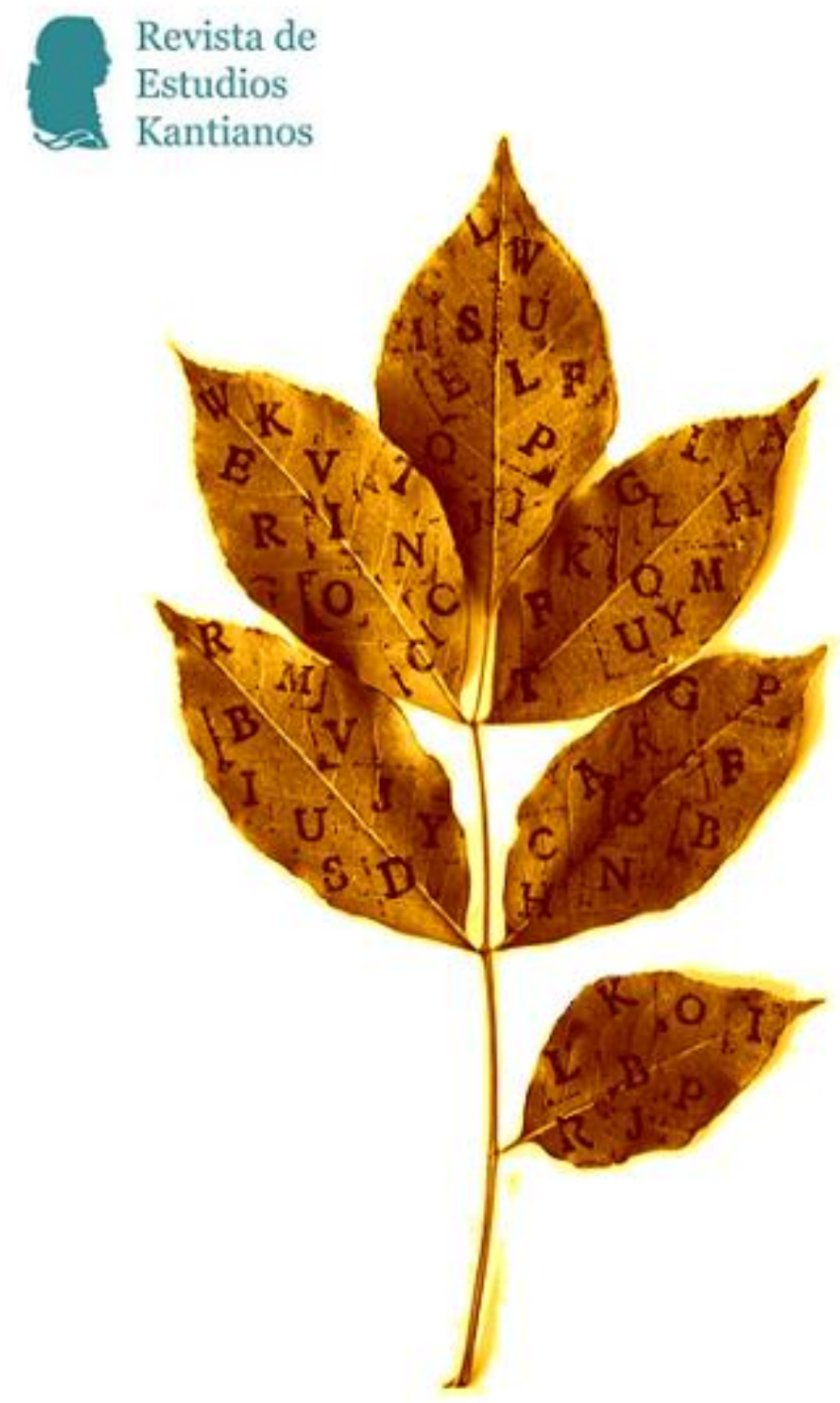


Revista de Estudios Kantianos 


\section{Revista de Estudios Kantianos}

Publicación internacional de la Sociedad de Estudios Kantianos en Lengua Española Internationale Zeitschrift der Gesellschaft für Kant-Studien in Spanischer Sprache International Journal of the Society of Kantian Studies in the Spanish Language

\section{Dirección}

Pedro Jesús Teruel, Universitat de València

pedro.teruel@uv.es

Hernán Pringe, CONICET-Universidad de Buenos Aires/

Universidad Diego Portales, Santiago de Chile

hpringe@gmail.com

\section{Secretario de edición}

Fernando Moledo, Universidad de Buenos Aires - CONICET

fernandomoledo@filo.uba.ar

\section{Secretaria de calidad}

Marcela García, Universidad de Morelia, México

garciar.marcela@gmail.com

\section{Editores científicos}

Jacinto Rivera de Rosales, UNED, Madrid

Claudia Jáuregui, Universidad de Buenos Aires

Vicente Durán, Pontificia Universidad Javeriana, Bogotá

Julio del Valle, Pontificia Universidad Católica del Perú, Lima

Jesús Conill, Universitat de València

Gustavo Leyva, Universidad Autónoma de México, México D. F.

María Xesús Vázquez Lobeiras, Universidade de Santiago de Compostela

Wilson Herrera, Universidad del Rosario, Bogotá

Pablo Oyarzun, Universidad de Chile, Santiago de Chile

Paula Órdenes Azúa, Universität Heidelberg 


\section{Comité científico}

Juan Arana, Universidad de Sevilla

Reinhardt Brandt, Philipps-Universität Marburg

Mario Caimi, Universidad de Buenos Aires

Monique Castillo, Université de Paris-Est

Adela Cortina, Universitat de València

Bernd Dörflinger, Universität Trier

Norbert Fischer, Universität Eichstätt-Ingolstadt

Miguel Giusti, Pontificia Universidad Católica del Perú

Dulce María Granja, Universidad Nacional Autónoma de México

Christian Hamm, Universidad Federal de Santa María, Brasil

Dietmar Heidemann, Université du Luxembourg

Otfried Höffe, Universität Tübingen

Claudio La Rocca, Università degli Studi di Genova

Juan Manuel Navarro Cordón, Universidad Complutense, Madrid

Carlos Pereda, Universidad Nacional Autónoma de México

Gustavo Pereira, Universidad de la República, Uruguay

Ubirajara Rancan de Azevedo, Universidade Estadual Paulista, Brasil

Margit Ruffing, Johannes Gutenberg-Universität Mainz

Gustavo Sarmiento, Universidad Simón Bolívar, Venezuela

Sergio Sevilla, Universitat de València

Roberto Torretti, Universidad Diego Portales, Santiago de Chile

Violetta Waibel, Universität Wien

Howard Williams, University of Aberystwyth

Allen W. Wood, Indiana University

Diseño, revisión de estilo y maqueta

Josefa Ros Velasco, Harvard University, Cambridge (MA)

\section{Corrector}

Aldo Perán, Universidad Diego Portales, Santiago de Chile

\section{Entidades colaboradoras}

Sociedad de Estudios Kantianos en Lengua Española (SEKLE)

Departament de Filosofia de la Universitat de València

Instituto de Humanidades, Universidad Diego Portales

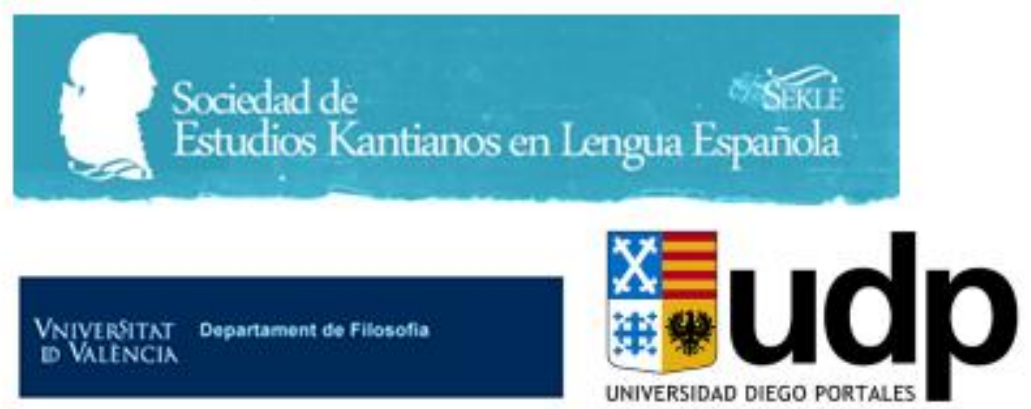




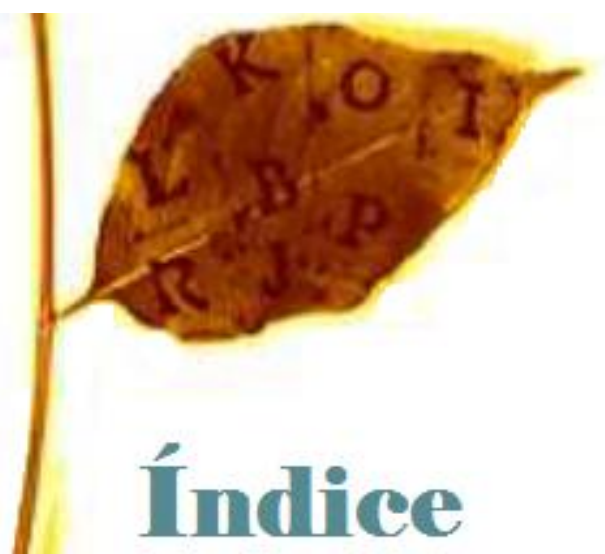

\section{Artículos}

125 Los deberes del amor en la doctrina kantiana de la virtud Bernd Dörflinger DOI 10.7203/REK.2.2.10807

135 Towards a new conception of metaphysics: Lambert's criticism on Wolff's mathematical method

Gesa Wellmann

DOI 10.7203/REK.2.2.10724

149 Acerca del alcance objetivo de las prescripciones metodológicas de la función regulativa de la razón teórica en la Crítica de la razón pura de Kant

Martín Arias Albisu

DOI 10.7203/REK.2.2.8432

168 Formas de autoconciencia en la "Reflexión de Leningrado" Matías Hernán Oroño

DOI 10.7203/REK.2.2.8799

179 Kants Konzeption kosmologischer Freiheit - ein metaphysischer Rest?

Christian Krijnen

DOI 10.7203/REK.2.2.10407

\section{Informe}

196 VII Coloquio Multilateral-Kant 2017

Paula Órdenes Azúa

DOI 10.7203/REK.2.2.10756 


\section{Reseñas}

200 Alfredo Ferrarin: Il pensare e l'io. Hegel e la critica di Kant. Roma, Carocci Editore, 2016, 244 pp. ISBN: 978-88-430-8246-9.

Luigi Filieri

DOI 10.7203/REK.2.2.10764

205 Mario Caimi, Ileana Beade, José González Ríos, Macarena Marey, Fernando Moledo, Mariela Paolucci, Hernán Pringe y Marcos Thisted: Diccionario de la filosofía crítica kantiana. Buenos Aires, Colihue, 2017, 507 pp. ISBN: 978-950-563-450-7.

Miguel Herszenbaun

DOI 10.7203/REK.2.2.10499

210 Paula Órdenes Azúa y Daniela Alegría: Kant y el Criticismo: pasado, presente, y ¿futuro?, Porto Alegre, Editora Fi, 2015, 303 pp. ISBN: 978-85-66923-52-0.

Constanza Terra

DOI 10.7203/REK.2.2.10777

212 Juan Ormeño Karzulovic y Miguel Vatter (eds.): Forzados a ser libres. Kant y la teoría republicana del derecho. Santiago, Fondo de Cultura Económica, 2017, 243 pp. ISBN: 978956-289-149-3.

Cristóbal Olivares

DOI 10.7203/REK.2.2.10762

215 Immanuel Kant (ed. y trad. Gonzalo Serrano): La Deducción trascendental y sus inéditos, 1772-1788. Bogotá, Universidad Nacional de Colombia, 2014, 321 pp. ISBN: 978-95-876195-39.

Pedro Stepanenko

DOI 10.7203/REK.2.2.10763

\section{Semblanza}

220 Juan Adolfo Bonaccini (16 de mayo de 1965 / 10 de julio de 2016)

Daniel Omar Perez

DOI 10.7203/REK.2.2.10783

\section{Convocatorias y normas para autores}

223 Kant en México

DOI 10.7203/REK.2.2.10863

224 IV Congreso Internacional de la SEKLE, Valencia, 2018

DOI 10.7203/REK.2.2.10863

227 VIII Coloquio Multilateral-Kant 2018

DOI 10.7203/REK.2.2.10863

229 Normas para autores

DOI 10.7203/REK.2.2.10863 


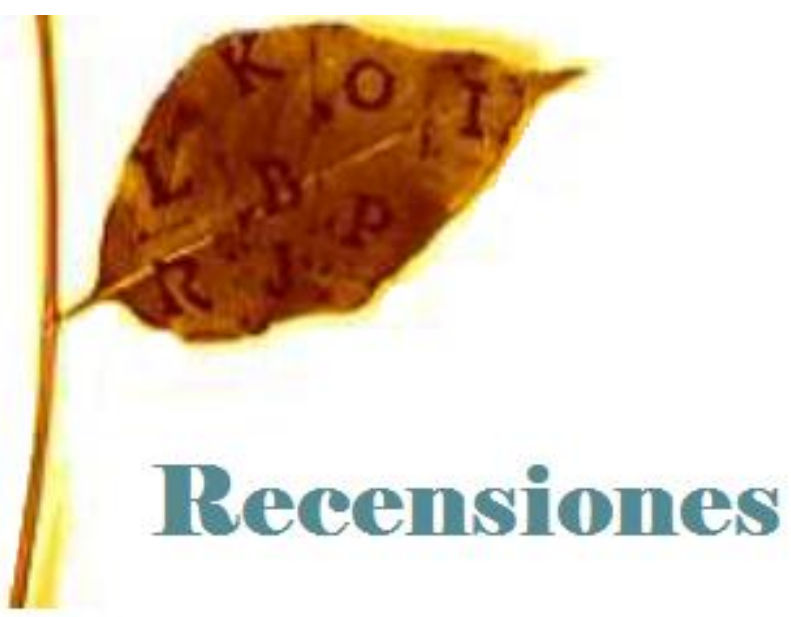




\title{
Mario Caimi, Ileana Beade, José González Ríos,Macarena Marey, Fernando Moledo, Mariela Paolucci, Hernán Pringe y Marcos Thisted: Diccionario de la filosofía crítica kantiana, Buenos Aires, Colihue, 2017, 507 pp. ISBN 978-950-563-450-7
}

\author{
Miguel HeRSZENBAUN ${ }^{1}$
}

\begin{abstract}
El texto de referencia es el esfuerzo conjunto de un grupo de investigadores argentinos especializados en la filosofía de Kant y la filosofía moderna. El coordinador del proyecto es Mario Caimi, por muchos años profesor titular dela materia Historia de la Filosofía Moderna en la Facultad de Filosofía y Letras de la Universidad de Buenos Aires, hoy profesor consulto de dicha casa de estudios, e investigador superior del Consejo Nacional de Investigaciones Científicas, Experimentales y Técnicas (CONICET)
\end{abstract}

Los autores restantes son reconocidos profesores de diversas instituciones universitarias argentinas (Universidad de Buenos Aires y Universidad Nacional de Rosario) y en su mayoría investigadores del CONICET. Se trata de un grupo que reúne especialistas en diversas áreas de la filosofía de Kant y en filosofía moderna en general. Casi todos ellos pertenecen al Grupo de Estudios Kantianos, grupo de investigación radicado en el Instituto de Filosofía Dr. Alejandro Korn de la Facultad de Filosofía y Letras de la Universidad de Buenos Aires.

El mencionado libro es el resultado de más de diez años de investigación. Se trata de un diccionario enciclopédico que recorre las diversas áreas de la filosofía kantiana y ofrece una mirada profunda y abarcadora sobre ellas. Como explica su prólogo, el Diccionario toma en cuenta las reconocidas obras de Mellin (Encyclopädisches Wörterbuch der kritischen Philosophie) y de Eisler (Kant Lexikon), pero no se trata de su traducción, sino que cada entrada es el resultado de la labor de los autores. Cabe mencionar que cada entrada va acompañada de su equivalente en alemán, latín, francés, italiano y portugués.

A fin de ejemplificar la profundidad y claridad del Diccionario, conviene detenerse en algunas de sus entradas.

Tomemos, por ejemplo, el término 'antinomia'. Los autores comienzan por indicar los múltiples sentidos que posee el término: en sentido objetivo, se refiere a la oposición de dos juicios que tienen una pretensión a priori de universalidad; en sentido subjetivo, se trata del estado de la razón del que, en busca de la totalidad absoluta, surgen raciocinios dialécticos que llevan a juicios contrapuestos. Estos juicios son los que conforman la antinomia en sentido objetivo. Los autores aclaran que el conflicto de estos juicios "es sólo aparente, pero esa apariencia de conflicto es inevitable" (p. 38).

Los autores señalan que contamos con tres especies de antinomia: la antinomia de la razón especulativa, la antinomia de la razón práctica y la antinomia de la razón con respecto a la facultad de juzgar. La antinomia de la razón especulativa se presenta bajo la forma de cuatro antinomias clasificadas según la tabla de las categorías. Estas se dividen en antinomias matemáticas (las dos primeras) y antinomias dinámicas (las dos últimas). La primera antinomia se refiere al origen y límites del mundo; la segunda se refiere a la divisibilidad o simplicidad de sus partes; la tercera se refiere a la posibilidad o imposibilidad de la causalidad por libertad; la cuarta se refiere a la existencia o inexistencia de un ente necesario.

${ }^{1}$ CONICET - Universidad de Buenos Aires. 
Luego de presentar los conflictos cosmológicos de la Crítica dela razón pura, los autores pasan a una explicación sistemática de cómo son resueltos. En las cuatro antinomias, la contradicción entre los enunciados resulta irresoluble, si se sostiene que el mundo sensible es una cosa en sí. Por el contrario, una vez aceptado que el mundo es mero fenómeno, las contradicciones desaparecen. En el caso de las antinomias matemáticas, una vez aceptado que el mundo es mero fenómeno, se concluye que los juicios enfrentados son falsos. En el caso de las antinomias dinámicas, la distinción entre un mundo fenoménico y un mundo inteligible permite admitir que los pares de juicios son verdaderos, sólo que las tesis lo serán en relación con el mundo inteligible y las antítesis con el mundo sensible. La antinomia de la razón pura vale como una prueba indirecta de la idealidad transcendental de los fenómenos: si el mundo fuera una totalidad existente en sí, nos veríamos conducidos necesariamente a una contradicción; por lo tanto, debemos admitir que el mundo es fenoménico.

La antinomia de la razón práctica se presenta en las esferas de la ética y del derecho. La antinomia de la razón práctica en la esfera de la ética emerge del concepto de sumo bien. "El sumo bien es la síntesis necesaria de felicidad y virtud. Pero ni la apetencia de felicidad puede ser el móvil que nos lleve a buscar la virtud, ni la virtud es la causa eficiente de la felicidad, por lo que la unión de felicidad y virtud parece imposible" (p.39). Conforme a la distinción entre mundo sensible y mundo inteligible ha de encontrarse la solución de esta antinomia. La primera proposición — que la apetencia de la felicidad sea el móvil que nos conduce a la virtud - es falsa. La segunda proposición - i.e. que la virtud sea la causa de la felicidad-, en cambio, sólo es falsa cuando se la entiende como forma de la causalidad en el mundo sensible. Ahora bien, en tanto puedo pensarme como noúmeno en el mundo inteligible, puedo admitir, en este respecto, como posible que la intención moral tenga una conexión causal necesaria con la felicidad. Así, puedo pensar la posibilidad de la unión necesaria de virtud y felicidad en el mundo inteligible. Finalmente, en tanto estamos obligados a perseguir el sumo bien, debemos necesariamente suponer a la virtud como causa de la felicidad.

La antinomia de la facultad de juzgarse encuentra en el uso estético y en el uso teleológico de la facultad de juzgar. La primera enfrenta dos juicios: uno que sostiene que el juicio del gusto no se basa en conceptos y otro que afirma que el juicio del gusto se basa en conceptos. La solución consiste en admitir que ambos juicios son verdaderos, pero que el término 'concepto' se entiende en diferentes sentidos. La antinomia del uso teleológico de la facultad de juzgar presenta una contraposición entre un juicio que afirma que la producción de toda cosa material debe ser juzgada como posible por medio de leyes meramente mecánicas y otro juicio que sostiene que ciertos productos (los organismos) no pueden ser producidos sólo por medio de estas leyes. Los autores explican que se trata de un conflicto aparente, pues aquí no se atiende a la constitución de las cosas, sino al modo en que debemos juzgarlas. Así, todo fenómeno debe ser explicado según leyes mecánicas. Pero, a la vez, los organismos deben ser juzgados como si estuvieran bajo algún principio que actuase de acuerdo con causas finales.

Finalmente, los autores arriban a una antinomia de la razón práctico-jurídica sobre el concepto de lo propio y de lo ajeno. Esta antinomia enfrenta un juicio que sostiene que es posible tener algo externo, aunque no se esté en posesión actual de ello y otro juicio que sostiene que no se puede tener algo externo como propio si no se está en posesión de ello. La solución se halla en la distinción entre una posesión empírica (a la que se refiere la tesis) y una posesión inteligible (a la que se refiere la antítesis).

Pasemos al término 'razón'. A este término, de evidente importancia en la obra de Kant, se le dedican cinco páginas. Se lo estudia desde el punto de vista lógico, gnoseológico, transcendental, metafísico y antropológico. A su vez, se considera a la razón en su uso teórico como en su uso práctico.

En lo que refiere a su uso teórico (en el que la facultad determina su objeto), la razón en su uso lógico es la facultad de deducir lo particular de lo universal. Se trata de la facultad capaz de llevar a cabo raciocinios (silogismos) o inferencias mediatas. Desde el punto de vista gnoseológico y según este mismo uso teórico, se la concibe como facultad de los principios: llevar a cabo un silogismo es alcanzar un conocimiento a partir de otros conocimientos, utilizando la premisa mayor del silogismo como un principio para ello. Por medio de este procedimiento, la razón procura llevar los múltiples conocimientos del entendimiento a una unidad sistemática a priori, ordenando las múltiples reglas del entendimiento bajo la unidad de principios superiores. La representación de esta unidad se da bajo 
representaciones denominadas ideas, que no brindan principios constitutivos de objetos, sino principios meramente regulativos de la actividad del entendimiento. Cuando a estas representaciones se les atribuye validez objetiva (i.e. se las toma como principios constitutivos de objetos), se producen los raciocinios dialécticos estudiados en la 'dialéctica transcendental'. Aquí entramos en el terreno del uso transcendental (ilegítimo) de la razón, en tanto esta facultad es utilizada como facultad del conocimiento puro, que pretende ilegítimamente ampliar el conocimiento a priori más allá de los límites de la experiencia. Por medio de su uso lógico, a través de tres tipos de prosilogismo (silogismo ascendente), la razón nos conduce a tres representaciones de lo incondicionado o ideas: Alma, Mundo y Dios. Aquí se trata de la razón como facultad de las ideas o de la razón en su uso real, a diferencia del mero uso lógico formal. Estas ideas o representaciones de lo incondicionado no pueden tener un objeto correspondiente en la experiencia. Por esto, puede decirse que la razón, en sentido metafísico y como facultad de lo incondicionado, realiza un esfuerzo por obtener un conocimiento de lo suprasensible. A pesar de que el conocimiento teórico de lo incondicionado le está vedado, ella es capaz de un conocimiento teórico simbólico y analógico.

Pasemos a considerar la razón en su uso práctico. La razón es 'práctica' en tanto se refiere a su objeto para darle efectiva existencia. Se trata de la facultad que determina el hacer y el obrar. Es razón práctica 'técnica' cuando los determina según fines contingentes sensibles. Aquí la facultad brinda sólo imperativos condicionados, destinados a alcanzar fines contingentes. Si, por el contrario, la razón determina el querer y el obrar por medio de puros conceptos, se trata de la razón 'práctica pura'. Esta es la "facultad de determinar por medio de ideas (conceptos racionales puros) el uso libre de nuestra causalidad" (p. 403). A esto llama Kant en los seres humanos el principio de autonomía o de libertad.

La razón práctica pura contiene principios a priori constitutivos válidos para la facultad apetitiva, por medio de lo cual ella determina lo que debe ser. Es la razón práctica pura la fuente de una norma absoluta, que al hacérsenos consciente - a través de lo que se denomina factum de la razón, esto es, "un hecho suprasensible en el sujeto moral" (p. 403) - conocemos la causalidad suprasensible en el ser humano como ser racional (o causalidad por libertad). Esto nos ofrece un conocimiento práctico (no teórico) de un ente con causalidad no sensible y nos conduce a los postulados de la razón (libertad de la voluntad, inmortalidad del alma y existencia de Dios), cuya realidad objetiva es sólo práctica. Es por medio del imperativo categórico como legislación de la razón práctica pura como el ser humano se descubre como miembro legislador de un reino inteligible de los fines y como un fin en sí mismo. El objeto de la razón práctica pura es el bien supremo. La razón en su uso práctico prima sobre la razón en su uso teórico. Esto significa que la razón proporciona la determinación práctica de lo que se encuentra fuera del alcance del uso teórico de esta facultad, ocupando el concepto de 'libertad' un lugar preponderante en el sistema crítico.

La entrada del Diccionario concluye abordando la razón en sentido antropológico, indicando que aquí se llama razón a la facultad de concebir por uno mismo los principios del conocimiento y de la acción, a lo que se opone la 'incapacidad legal' o 'minoridad'.

Veamos el término 'transcendental'. Los autores comienzan indicando un sentido del término 'transcendental' no presente expresamente en los textos kantianos. Se dice de aquellos predicados que se pueden enunciar con verdad de manera necesaria y universal de toda cosa. En este sentido, se trataría de predicados que están más allá de todo género y toda clasificación. Luego de la Revolución Copernicana, para Kant no habría predicados que estén por encima de las categorías, en tanto todo objeto, para serlo, depende de una síntesis categorial. Así, las categorías, como condiciones de objetividad de todo objeto, pueden ser llamadas 'transcendentales'. De esta manera, explican los autores, "se llaman [...] transcendentales aquellos predicados que se pueden enunciar de manera necesaria y universal (es decir, a priori) de todos los objetos porque son condiciones de la objetividad misma" (p. 475).

En la filosofía kantiana, el término transcendental se aplica a cierto conocimiento de segundo orden, al conocimiento del conocimiento a priori. El conocimiento transcendental pretende explicar cómo es posible conocer a priori. Se trata de un conocimiento del conocimiento a priori y de cómo éste es posible. Se trata, así, de una "teoría del conocimiento" (p. 476) que se ocupa de "la crítica del conocimiento" ( $\mathrm{KrVB} 81$; ápud p. 476). El conocimiento transcendental tiene por funciones propias fundamentar el conocimiento a priori y establecer el alcance y límite de las facultades 
cognoscitivas utilizadas en el conocimiento de objetos. También se califica de 'transcendental' a cada elemento identificado como condición a priori del conocimiento en general y a las condiciones del conocimiento a priori. Algo es transcendental si es fuente de fundamentación de la posibilidad de conocimiento a priori.

Los autores distinguen el conocimiento transcendental de las estructuras del pensamiento de la lógicaformal, del conocimiento transcendente, del conocimiento empírico y "del conocimiento a priori que no versa sobre otros conocimientos, sino sobre la sensibilidad o sobre objetos" (p. 476).

Primero, el conocimiento transcendental es un conocimiento del conocimiento, es decir, de pensamientos referidos a objetos, y se opone a las meras estructuras a priori del pensamiento construidas por la lógica formal (meros pensamientos no necesariamente referidos a objetos). La lógica deja de ser meramente formal y pasa a ser 'transcendental', cuando se ocupa de las reglas del pensamiento puro de objetos. Así, el término transcendental queda vinculado al 'conocer' y opuesto a lo lógico-formal, asociado al mero 'pensar'.

Segundo, los autores distinguen conocimiento transcendental de conocimiento transcendente (advirtiendo aquellos casos en que Kant utiliza el primer término en el sentido del segundo). El uso transcendental de conceptos se refiere al uso a priori respecto a objetos, el que sólo es legítimo en tanto se refiera a objetos dados en la sensibilidad, y no es legítimo si se pretende conocer a priori cosas en sí u objetos no sensibles, es decir, si se pretende conocer a priori objetos sin atenerse a las condiciones de la sensibilidad. Este uso transcendental ilegítimo es más exactamente un 'uso transcendente'. Ahora bien, "sólo la posibilidad de la experiencia sirve de fundamento de la validez del conocimiento transcendental" (p. 477), con lo cual todo uso de las categorías que no remita a la experiencia posible y a las condiciones formales de la sensibilidad (sólo dentro de cuyos límites puede ser dado el objeto al que refieren los conceptos puros del entendimiento) es ilegítimo y 'transcendente'. Es en este sentido como 'transcendental' termina por significar "relativo a la posibilidad de la experiencia" ( $K r V B$ 401, ápud p. 477).

Tercero, el conocimiento transcendental se relaciona con el conocimiento empírico, en tanto en éste se encuentran también elementos a priori. Es tarea del conocimiento transcendental explicar la posibilidad del conocimiento empírico, en tanto entre las condiciones de posibilidad del conocimiento empírico hay condiciones que valen a priori (i.e. con independencia de la experiencia).

Cuarto, en lo que se refiere a la relación entre conocimiento transcendental y otros conocimientos a priori, los autores explican que no todo conocimiento a priori es necesariamente transcendental. No todo conocimiento a priori - por ejemplo, la geometría - tiene por objeto otro conocimiento (no es conocimiento de la posibilidad del conocimiento a priori). "El conocimiento $a$ priori que se puede llamar transcendental es aquel por el cual conocemos que ciertas representaciones (intuiciones o conceptos) se pueden aplicar a priori a objetos de la experiencia [...] porque sirven de fundamento de la posibilidad de un conocimiento a priori" (p. 478).

Por otra parte, el término transcendental se refiere al origen del conocimiento, esto es, a las facultades que hacen posible el conocimiento a priori. En este sentido, la unidad sintética necesaria del múltiple fenoménico y la imaginación son transcendentales, en tanto sólo gracias a ellas se produce la experiencia. La síntesis transcendental no sólo tiene lugar a priori, sino que fundamenta otros conocimientos a priori.

Finalmente, el término transcendental se refiere a la filosofía transcendental, la cual es un sistema de todos los conocimientos transcendentales, cuya tarea es explicar cómo es posible el conocimiento a priori, i.e. explicar cómo son posibles los juicios sintéticos a priori (juicios a través de los cuales se vehiculiza dicho conocimiento). El examen de dicho conocimiento es la Crítica de la razón pura. "La filosofía transcendental es el sistema [...] de todos los conceptos y principios cuyo origen es a priori y que se refieren a objetos en general" (p. 479). Todo esto es asunto de la Crítica de la razón pura, que es la idea completa de la filosofía transcendental, pero no la ciencia misma, pues esta Crítica sólo alcanza la evaluación completa de los principios del conocimiento a priori. A esta Crítica también compete establecer los conceptos y principios de la filosofía transcendental, la explicación de su posibilidad y el trazado arquitectónico de todo su plan. 
El Diccionario recorre la totalidad de la obra kantiana y se ocupa de los términos científicamente relevantes y de otros de menor alcance; se detiene en un estudio sistemático y detallado de los conceptos fundamentales de la filosofía crítica, condensa lo fundamental de cada materia y lo expone de manera sistemática, ordenada, profunda y clara.

Es un libro de referencia obligatoria para especialistas en la obra de Kant, en el Idealismo alemán y en la filosofía moderna, así como para doctorandos y estudiantes de grado. Vale aclarar que se trata del primer diccionario kantiano en español, estableciéndose así como un excelente antecedente para la tradición de kantianos hispanoparlantes y que permanecerá en esta tradición como una referencia ineludible.

Celebro la aparición de este Diccionario y que venga de la mano de un grupo de especialistas en el que está muy bien representado el Grupo de Estudios Kantianos. 